\title{
Evaluation of remineralization potential of casein phospho-peptide with amorphous calcium phosphate fluoride (CPP-ACPF) on demineralised enamel surfaces.
}

\author{
Ali A Assiry* \\ Department of Preventive Dental Science, Faculty of Dentistry, Najran University, Najran, Saudi Arabia
}

\begin{abstract}
Dental caries is one of the worldwide common disease affecting both rural and urban communities. Demineralization is the loss of significant amount of minerals, especially calcium and phosphate from the tooth enamel leading to caries formation. The process of demineralization and remineralization is considered as a dynamic process, characterized by the flow of phosphate and calcium out of and back in the enamel. Therefore, the current in-vitro study was designed for evaluating the remineralization potential of casein phospho-peptide with amorphous calcium phosphate fluoride (CPP-ACPF) on the artificially demineralized enamel surfaces samples. Thirty enamel samples were subjected to artificial demineralization procedure followed by remineralization using CPP-ACPF for one week in ten samples, two weeks in next ten sample and four-week $s$ in last ten samples. The final results were analysed using the Confocal Laser Scanning Microscopic (CLSM) and Image $J$ software. The mean score of remineralization potential for one, two- and four-week samples was found to be $59.1064,58.96$ and 56.95 respectively. The mean gray value scores were almost similar in one, two- and four-week samples i.e., the remineralization potential does not increase or decrease significantly with increasing time. But the overall CPP-ACPF remineralization potential was high and therefore it can be used as a potential agent to prevent dental caries. The data from our current study will help to make clinical use of the agent as a potential and excellent local slow-delivery system to treat the white spot lesion in enamel.
\end{abstract}

Keywords: Dental Caries, Remineralization, Demineralization, CPP-ACPF, CLSM.

Accepted on June 29, 2019

\section{Introduction}

Dental caries is a common disease and a worldwide public health concern. It affects a huge population including several rural and urban communities [1]. One of the currently available review on epidemiological data of various countries evidently showed marked rise in the prevalence of dental caries [2]. The initial indications of early carious lesion is the chalky "white spot' appearance on the tooth surface. This appearance indicates an area of demineralization of enamel and referred as an incipient carious lesion or an early carious lesion [3]. The prevalence of dental caries in developing countries affects both the adults and children in the primary or permanent teeth, and root as well as coronal surfaces. Several strategies were adopted worldwide for the prevention of dental caries such as topical fluoride application, water fluoridation, a return to school oral health educational programs, proper diet and regular dental office visits, the use of fluoride rinses, focus on proper brushing with fluoride dentifrice, and also flossing [4].

The process of remineralization and demineralization is considered as a dynamic process, characterized by the flow of phosphate and calcium out of and back in the enamel. Also, the flow should be balanced to prevent the development of caries in enamel. It implies that the cavity formation can be prevented if the average amount of remineralization is equal or less than the demineralization. The concentration of phosphate and calcium in saliva and plaque fluid determines the $\mathrm{pH}$ at which remineralization and demineralization occurs [5]. There is a major requirement to enhance remineralization process by finding different ways and thus transfer such information in clinical therapy to modify caries balance, especially in people with high cariogenic bacterial challenge [6].

One such method of remineralization is calcium phosphate based remineralizing technique. This technique is more effective in remineralizing the early carious lesions when compared to the use of fluoride alone. Many products with calcium and phosphate are being evaluated or are available in the market for reversing or inhibiting caries. One such product is the CPP-ACP (casein phosphopeptide with amorphous calcium phosphate) complex [7]. The casein present in bovine milk is the predominant phosphoprotein, which makes $80 \%$ of the total protein. It primarily acts as a phosphate-calcium stabilized micellular complexes [8]. The sequence cluster of from casein, -Ser (P)-Ser (P)-Ser (P)-Glu-Glu, makes up the casein phosphopeptides (CPP) [2,8]. In the alkaline conditions, the phosphate-calcium exists in an alkaline amorphous state 
complexed with CPP and referred as CPP-ACP [9]. The CPP$\mathrm{ACP}$ readily binds to the tooth surface and the bacteria in present plaque. The main function of CPP-ACPF (CPP-ACP fluoride) cream is stabilization of high concentrations of phosphate and calcium ions at surface of tooth by binding at plaque and pellicle. It provides a very effective mode to elevate the amount of calcium in fluid of dental plaque, which is desirable for increasing the remineralization [10].

\section{Materials and Methods}

\section{Study design and of reagents preparation}

The present in-vitro study was planned for evaluating the remineralization potential of the remineralizing agent, CPPACPF, on artificially demineralized enamel surfaces.

The artificial saliva [Wet Mouth] [ICPA] and buffered demineralisation solution was prepared from the analyticalgrade chemicals and distilled water. The artificial saliva consists of sodium carboxy methyl cellulose $0.5 \% \mathrm{w} / \mathrm{v}$, glycerin $30 \% \mathrm{w} / \mathrm{v}$, water, paraben and pleasantly flavored base without any essential calcium and phosphate ions. The prepared demineralisation solution consists of $2.2 \mathrm{mM}$ Mono Potassium dihydrogen Phosphate $\left(\mathrm{KH}_{2} \mathrm{PO}_{4}\right), 2.2 \mathrm{mM}$ Calcium Chloride $(\mathrm{CaCl} 2)$ and $0.05 \mathrm{M}$ Acetic acid. The $1 \mathrm{M}$ Potassium hydroxide $(\mathrm{KOH})$ was used to adjust the $\mathrm{pH}$ to 4.4. Also, Rhodamine $\mathrm{B}$ dye $(0.01 \mathrm{mM})$ was prepared by adding 23.95 $\mathrm{mg}$ of Rhodamine-B into $500 \mathrm{ml}$ of distilled water. The remineralizing agent used for the evaluation was $\mathrm{CPP}-\mathrm{ACPF}$ $(0.2 \%$ or $900 \mathrm{ppm}$ of fluoride concentration) [GC Tooth Mousse Plus $\left.{ }^{\circledR}\right]$. The teeth with sound enamel and dentin without any structural defects and carious lesions were included for the study. Teeth with white spot lesions, initial cavitated lesions, cracks and other external structural defects were excluded from this study.

\section{Sample preparation and demineralization of enamel}

Fifteen maxillary premolars designated for orthodontic extraction were selected for the study. The teeth were cleaned thoroughly, and any type of debris were removed. It was then stored in normal saline until further use. Next, the roots of the teeth samples were dissected using a diamond disc and hand motor. The crown part of the teeth was sectioned mesio-distally into buccal and lingual halves to obtain 30 sectioned teeth samples. A window of " $4 \times 4 \mathrm{~mm}$ " was created by sticking the sticker of same size on the centre of enamel surfaces of sectioned samples. The remaining surfaces were coated with an acid resistant nail varnish and were allowed to dry for 6 hours. After drying, the stickers were removed, and the samples were subjected to demineralization procedure by placing each tooth sample in separate vials containing $4 \mathrm{ml}$ of demineralization solution for four days.

\section{Remineralization of the demineralized samples}

Each tooth sample from the study groups were sectioned labiolingually via the center of window in 2 equal halves. The first half served as the control group, the second half received the application of remineralizing agent and served as the experimental group. The experimental group received CPPACPF applications with the help of an applicator tip once daily for 4 minutes, followed by rinsing in distilled water and were stored in individual container with artificial saliva for 24 hours. This process was continued for 14 days $(4,10)$. After 14 days, these experimental group was stored in an individual container with artificial saliva for 1 week, 2 weeks and 4 weeks respectively. The artificial saliva was replaced every day (4, 17). At the end of Ist week (10 samples), IInd week (next 10 samples) and IVrth week (remaining 10 samples), the samples were subjected to CLSM evaluation to determine the remineralization potential.

\section{CLSM and statistical analysis}

The control and experimental group were subjected to Confocal Laser Scanning Microscopic (CLSM) evaluation to measure the baseline data of demineralization. The teeth samples from the control and experimental groups were kept in Rhodamine B dye for 24 hours. The stained sections then were placed on the microscopic glass slide with $10 \mathrm{X}$ objective, at $50 \%$ intensity of Argon laser illumination at $488 \mathrm{nM}$ excitation wavelength. With a $515 \mathrm{~nm}$ long-pass filter, the confocal slits were set at $25 \mu \mathrm{m}$. The cross-sectional analysis of enamel lesions and the remineralized samples with CLSM is based on the digital images taken at certain controlled settings. Analysis of all samples was done with a Carl-Zeiss CLSM. The accompanied software (Image J program) calculates imagebased parameters of selected lesion zones. The data obtained were subjected to statistical analysis. The statistical tests used in this study were mean, standard deviation and one-way ANOVA test.

\section{Results}

The present study was designed to evaluate the remineralization potential CPP-ACPF on demineralized enamel surfaces. Fifteen premolars samples, with sound enamel and free of structural defects, were collected and prepared and dissected into final 30 samples. The samples were subjected to demineralization process and then divided into two groups, control and experimental group. The experimental group were subjected to remineralization process with CPPACPF.

\section{Remineralization potential after one, two and four weeks}

The control and experimental sample were evaluated by CLSM for remineralization potential of the CPP-ACPF. The CLSM images of demineralized and remineralized teeth samples were evaluated using Image $\mathrm{J}$ software. The potential of the CPPACPF was observed after one, two and four weeks of remineralization procedure for three different experimental sets of samples (each set with 10 sample). A distinct remineralizing band was observed in the CLSM image for each group i.e., one, two and four week remineralized enamel sample. Figure 1 


\section{$A C P F)$ on demineralised enamel surfaces.}

showed the demineralized section and remineralized section of an enamel sample after one-week of remineralization using CPP-ACPF. The CLSM image clearly indicates a distinct remineralization band suggesting that CPP-ACPF successfully and efficiently remineralized the sample as compared to demineralized sample. Similarly, the CLSM image for twoand four-week sample in Figures 2 and 3 respectively also showed a distinct remineralization band indicating successfully remineralizing by CPP-ACPF of demineralized enamel surface.
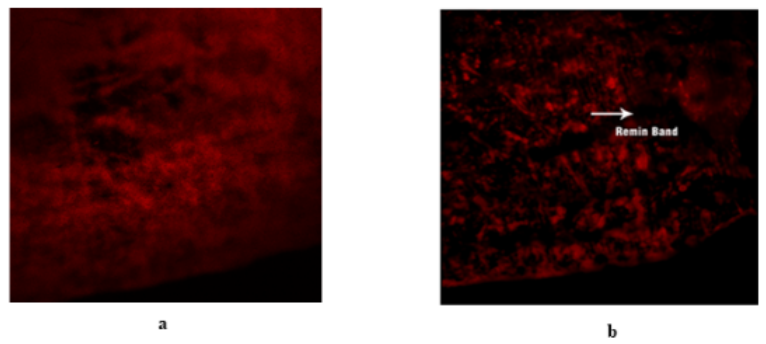

Figure 1. CLSM images of (a) the demineralized section and (b) the remineralized section of a tooth in one-week samples of $C P P-A C P F$.
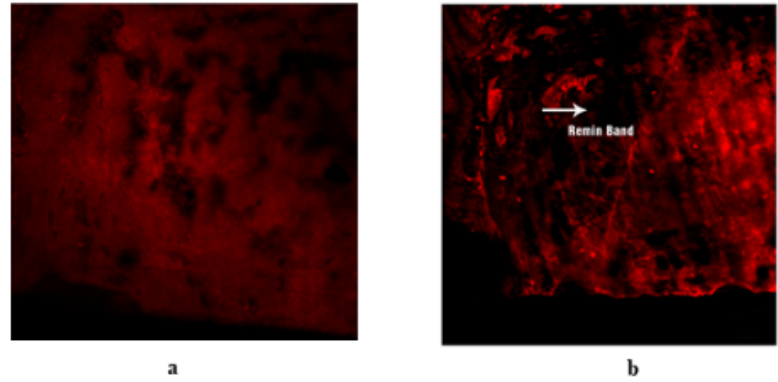

Figure 2. CLSM images of (a) the demineralized section and (b) the remineralized section of a tooth in two weeks samples of CPP-ACPF.
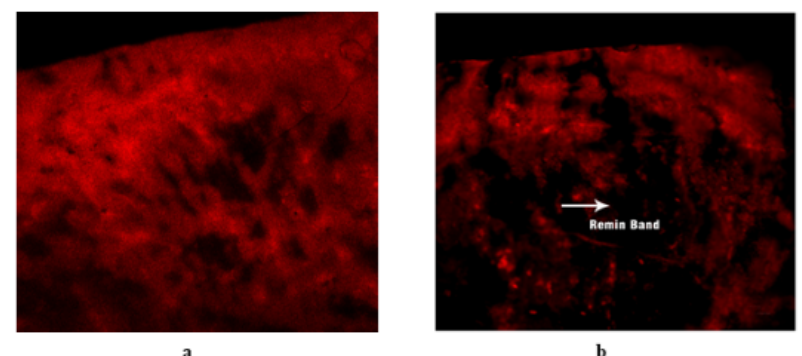

Figure 3. CLSM images of (a) the demineralized section and (b) the remineralized section of a tooth in four weeks samples of $C P P-A C P F$.

\section{Remineralization potential of CPP-ACPF}

It measured the mean gray value scores of the images and was numerically interpreted. The remineralization potential of each tooth sample was measured by calculating the difference between its baseline data (demineralized value) with its counterpart remineralized value. That is;

Remineralization potential=Demineralization mean gray value score - Remineralization mean gray value score
The analysis by CLSM for the untreated sample records a near zero fluorescence with grayscale value approximately zero and is pitch black. The demineralized sample or lesions slightly auto fluoresce but the imbibition of the $0.1 \mathrm{mM}$ Rhodamine dye allowed the porous demineralized layer (higher gray values) to fill and appear with significant contrast. The remineralized samples showed a reduced fluorescence (smaller gray values), which indicates less porosity and dye penetration or more mineral concentration $[1,11]$.

The remineralization potential mean gray value scores for oneweek samples ranged from 32.644 to 71.462 with mean score of 59.1064. In two weeks samples it ranged from 47.42 to 74.145 with mean score 58.96. In four weeks samples it ranges from 11.006 to 71.721 with mean 56.95 . The mean gray value scores were almost similar for one, two- and four-week samples i.e., the remineralization potential does not increase or decrease significantly with increasing time (Tables 1 and 2).

Table 1. Remineralization potential mean gray value scores of $C P P$ $A C P F$.

\begin{tabular}{llll}
\hline S. No. & 1 Week samples & 2 Weeks samples & 4 Weeks samples \\
\hline 1 & 47.995 & 74.145 & 61.27 \\
\hline 2 & 32.644 & 63.266 & 71.721 \\
\hline 3 & 69.572 & 55.415 & 11.006 \\
\hline 4 & 65.009 & 63.701 & 55.76 \\
\hline 5 & 64.904 & 61.685 & 62.419 \\
\hline 6 & 71.462 & 47.42 & 58.601 \\
\hline 7 & 62.623 & 51.973 & 63.332 \\
\hline 8 & 47.995 & 62.225 & 65.77 \\
\hline 9 & 64.97 & 53.996 & 59.419 \\
\hline 10 & 63.89 & 55.78 & 60.281 \\
\hline
\end{tabular}

Table 2. Descriptive statistics with mean and standard deviation.

\begin{tabular}{llll}
\hline & Week 1 & Week 2 & Week 4 \\
\hline $\mathrm{N}$ & 10 & 10 & 10 \\
\hline Mean & 59.1064 & 58.9606 & 56.9579 \\
\hline $\mathrm{SD}$ & 12.2359 & 14.5939 & 16.7248 \\
\hline
\end{tabular}

\section{Comparison of remineralization potential within the group}

The one-way ANOVA used for statistical analysis, was tested to determine whether any of the differences among the means are statistically significant. The calculated p-value, 0.915 , was found to be greater than significance level (0.05) (Table 3). The analysis revealed that there is no statistically significant difference between the mean gray value response of the one, two- and four-weeks of CPP-ACPF remineralized sample. Thus indicating that there is no significant difference in the 
remineralization potential of one, two- and four-weeks samples.

Table 3. Comparison of remineralization potential within the CPP$A C P F$ remineralized samples

\begin{tabular}{lllllll}
\hline GROUP-I & Mean & Df & SS & MSS & f-value & p-value \\
\hline $\begin{array}{l}\text { 1-week } \\
\text { samples }\end{array}$ & 59.1064 & 2 & 29 & 14 & 0.089 & 0.915 \\
\hline $\begin{array}{l}\text { 2-week } \\
\text { samples }\end{array}$ & 58.9606 & & & & & \\
\hline $\begin{array}{l}\text { 4-week } \\
\text { samples }\end{array}$ & 56.9579 & & & & & \\
\hline
\end{tabular}

\section{Discussion}

Remineralization is described as a process in which phosphate and calcium ions are supplied to the tooth via an external source. It promotes deposition of ion in the crystal voids in demineralized enamel for creating net gain of minerals. The word 'void' is defined as any available area in a crystal created by loss of ion from demineralization. Thus, remineralization can also be defined as crystal repair for creating net mineral gain on an enamel subsurface lesion. But it does not extend for precipitation of solid phases on enamel surfaces [12]. Many studies attempted to investigate several calcifying solutions as remineralizing agent on demineralized tooth surface or structure [13-18].

The fundamental issue in the clinical applications of phosphate and calcium remineralization system is the less solubility of calcium-phosphates, especially in the presence of fluoride ions. The single, low-concentration calcium-phosphate based remineralization system is not effective in localization of ions on the surface of tooth in substantial amount for promoting remineralization of enamel subsurface. This leads to the development or advancement of novel delivery systems based on calcium-phosphate, having high concentrations of phosphate and calcium. One such new product applying this type of delivery system is now commercially available as stabilized amorphous tooth cream known as CPP-ACPF. The manufacturer of these products claims that they provide a new avenue for remineralizing the non-cavitated caries lesions.5 This initiated the present in-vitro study for evaluating the remineralization potential of new commercially available calcium-phosphate-based remineralizing agent, CPP-ACPF, on demineralised enamel surfaces after storing in artificial saliva for periods of one, two and four weeks.

In the present study CPP-ACPF showed remineralization potential against the artificially demineralized enamel. The result was in agreement with the other previous studies on CPP-ACPF showing their remineralization potential on artificially induced initial enamel carious lesions [1,4,5,7,8,11,13-15,17,19-24]. Bhatt et al., showed in his study that CPP-ACPF exhibited significant amount of remineralization potential which was statistically significant at 21 days. 4 In our study the remineralization potential of CPP$\mathrm{ACPF}$, decreased gradually from one-week samples to four weeks samples and the difference or decrease in this potential was not statistically significant. The possible reasons for this result may be the action of CPP-ACPF molecules which binds at the position inside the lesion of enamel subsurface and at the surface of lesion. Once it is inside the enamel subsurface lesion, CPP-ACPF releases weakly bound fluoride, phosphate, and calcium ions, which then deposits in the crystal voids. The phosphate and calcium ions release is thermodynamically driven. The CPPs with high binding affinity for apatite; upon entering the lesion, may bind to more thermodynamically favorable surface of appetite crystal face. Henceforth, once the CPPs binds to an appetite crystal in the enamel subsurface lesion, it may play a significant role in regulating anisotropic crystal growth and also inhibition of crystal demineralization [5].

When low concentration of fluoride is provided to CPP-ACP, the formation of mineral in lesion is consistent with fluorhydroxyapatite or fluorapatite [5]. With low fluoride concentration use, as in CPP-ACPF, there is a complex localization of free fluoride, phosphate and calcium ion activities. It helps to maintain a state of supersaturation, by suppressing the demineralization process [13]. Therefore, in our study we can assume that the release of the calcium, phosphate and fluoride ions was maximum in the 1st week which then slightly decreases in the subsequent 2 nd and 4 th week samples. Thus, we can conclude that CPP-ACPF can act as an excellent local slow-delivery system to treat the white spot lesion in tooth.

\section{Conclusion}

In conclusion, the remineralization potential of CPP-ACPF was found to be maximum in the first week after the artificial demineralization process. It then slightly decreases in the subsequent weeks therefore it is not time dependent. Thus CPP-ACPF can be used as a local slow-delivery system efficiently to treat the white spot lesion in enamel.

\section{References}

1. Alaudin SS, Fontana M. Evaluation of Novamin ${ }^{\circledR}$ as an adjunct to fluoride for caries

2. lesion remineralization. NovaMin Research Report 2006.

3. Azarpazhooh A1, Limeback H. Clinical efficacy of casein derivatives: a systematic review of the literature. J Am Dent Assoc 2008; 139: 915-924.

4. Bagramian RA, Garcia-Godoy F, Volpe AR. The global increase in dental caries. A pending public health crisis. Am J Dent 2009; 22:3-8.

5. Bhat S, Hegde K, Habibullah M, Bernhardt V. Incipient enamel lesions remineralization using casein phosphopeptide amorphous calcium phosphate cream with and without fluoride: a laser fluorescence study. J Clin Pediatr Dent 2012; 36: 253-355.

6. Cochrane NJ1, Cai F, Huq NL, Burrow MF, Reynolds EC. New approaches to enhanced remineralization of tooth enamel. J Dent Res 2010; 89: 1187-1197. 


\section{$A C P F)$ on demineralised enamel surfaces.}

7. Featherstone JD1. Remineralization, the natural caries repair process--the need for new approaches. Adv Dent Res 2009; $21: 4-7$.

8. Golpayegani MV, Sohrabi A, Biria M, Ansari G. Remineralization effect of topical NovaMin versus sodium fluoride $(1.1 \%)$ on caries-like lesions in permanent teeth. J Dent (Tehran) 2012; 9: 68. Gonzalez-Cabezas C, Fontana M, Dunipace A, Li Y, Fischer G, Proskin H, Stookey G. Measurement of enamel remineralization using microradiography and confocal microscopy. Caries Res 1998; 32: 385-392.

9. Grewal H, Verma M, Kumar A. Prevalence of dental caries and treatment needs amongst the school children of three educational zones of urban Delhi, India. Indian J Dent Res 2011; 22: 517.

10. Hegde MN, Moany A. Remineralization of enamel subsurface lesions with casein phosphopeptide-amorphous calcium phosphate: A quantitative energy dispersive X-ray analysis using scanning electron microscopy: An in vitro study. J Conserv Dent 2012; 15: 61.

11. Holler B, Friedl K-H, Jung H, Hiller K-A, Schmalz G. Fluoride uptake and distribution in enamel and dentin after application of different fluoride solutions. Clin Oral Investig 2002; 6: 137-144.

12. Jamieson LM1, Thomson WM, McGee R. Caries prevalence and severity in urban Fijian school children. Int J Paediatr Dent 2004; 14: 34-40.

13. Jayarajan J, Janardhanam P, Jayakumar P. Efficacy of CPP$\mathrm{ACP}$ and $\mathrm{CPP}-\mathrm{ACPF}$ on enamel remineralization-An in vitro study using scanning electron microscope and DIAGNOdent ${ }^{\circledR}$. Indian J Dent Res 2011; 22: 77.

14. Kamath P, Nayak R, Kamath SU, Pai D. A comparative evaluation of the remineralization potential of three commercially available remineralizing agents on white spot lesions in primary teeth: An in vitro study. J Indian Soc Pedod Prev Dent 2017; 35: 229.

15. Madan N, Madan N, Sharma V, Pardal D, Madan N. Tooth remineralization using bio-active glass-A novel approach. J Acad Adv Dent Res 2011; 2: 45-50.

16. Mohanty B, Dadlani D, Mahoney D, Mann A. Characterizing and identifying incipient carious lesions in dental enamel using micro-Raman spectroscopy. Caries Res 2013;47: 27-33.

17. Patil N, Choudhari S, Kulkarni S, Joshi SR. Comparative evaluation of remineralizing potential of three agents on artificially demineralized human enamel: An in vitro study. J Conserv Dent 2013; 16: 116.

18. Pradeep K, Rao P. Remineralizing agents in the noninvasive treatment of early carious lesions. Int J Dent Case Reports 2011; 1: 73-84.

19. Preethee T, Kandaswamy D, Rosaline H, Arathi G. Comparing the remineralising potential of novamin and casein phosphopeptide-amorphous calcium phosphate using quantitative light induced fluorescence. Amrita J Med 2011; 7: 1-44.

20. Reynolds E, Cai F, Cochrane N, Shen P, Walker G, Morgan M, Reynolds C. Fluoride and casein phosphopeptideamorphous calcium phosphate. J Dent Res 200887: 344-348.

21. Shen P, Cai F, Nowicki A, Vincent J, Reynolds E. Remineralization of enamel subsurface lesions by sugarfree chewing gum containing casein phosphopeptideamorphous calcium phosphate. J Dent Res 2001; 80: 2066-2070.

22. Torrado A, Valiente M, Zhang W. Remineralization Potential of a New Toothpaste Formulation: An Vitro. J Contemp Dent Pract 2004; 15: 18-030.

23. Walsh LJ. Contemporary technologies for remineralization therapies: A review. Int Dent SA 2009; 11: 6-16.

24. Wefel J. NovaMin ${ }^{\circledR}$ : likely clinical success. Adv dental Res 2009; 21: 40-43.

\section{${ }^{*}$ Correspondence to}

Ali A Assiry

Department of Preventive Dental Science

Najran University

Saudi Arabia 